\title{
Johann Hochhuth and his collection of silken-fungus beetles (Coleoptera: Cryptophagidae): significance and unique specimens
}

\section{Kateryna Ocheretna}

\author{
Institute of Zoology NAS of Ukraine (Kyiv, Ukraine)
}

\begin{abstract}
Johann Hochhuth and his collection of silken-fungus beetles (Coleoptera: Cryptophagidae): significance and unique specimens. - K. Ocheretna. - Johann Hochhuth was one of the most significant amateur collectors and naturalists of the 19th century in Ukraine and other countries of Eastern Europe. His collection of Coleoptera has an immense amount of scientific material which is actual even nowadays. Cryptophagidae in Johann Hochhuth's collection is only a small but significant part of collection which can be used as a comparative material for newer gatherings. The author provides some historical facts about the collector and a detailed description of his silken-fungus beetles collection. This collection consists of 214 Cryptophagidae specimens belonging to 50 species of the family. The author also revised all of the specimens, clarified the identification of the species, updated the identification or species names if they weren't revised earlier, and indicated the most unique specimens of the collection. The collection includes only specimens from the territory of Kyiv and Kyiv Oblast, and some specimens collected by other collectors, such as Wilhelm Rosengauer (Austria and Germany), Christian Suffrian (Germany), Ernst von Ballion (Russia), Oleksandr Czekanowski (Ukraine) and others. The collection is undoubtedly important for the study of morphological pecularities of some rare species of the Cryptophagidae family compared to recently collected specimens. The accumulation of the information about the silken-fungus beetle fauna of Ukraine will make it possible to generalise the list of species in the studied region. This entomological collection is part of the history of the formation of modern large zoological collections through the difficult path from J. H. Hochhuth's collection, the part of M. Cherkunov's collection, the natural history collections of the Pedagogical Museum of Kyiv to the collections of the National Museum of Natural History, NAS of Ukraine. Its materials are a complete composition of Cryptophagidae species that were collected in the territory of Kyiv and Kyiv Oblast in the middle of the 19th century. This article also contains important biographical information on Johann Heinrich Hochhuth's life, education, and scientific achievements.
\end{abstract}

Key words: Cryptophagidae, Hochhuth, natural history museums, museum collections, Ukraine.

\section{Introduction}

Scientists and naturalists of the 19th and early 20th centuries were a special community of people who are often unrelated to any educational or scientific institution, such as universities or high schools, as one of the few centres of prosperity of scientific thought. Even though most naturalists were not professional zoologists, their contribution to the scientific research is often expressive. It should also be noted that a large number of natural collections, including entomological collections deposited in natural history museums of the world, were collected by amateur scientists who did not have titles or positions, but with great enthusiasm engaged in scientific research (Ocheretna, $2019 c$ ). These statements can be applied to the weighty figure of Kyiv of the 19th century - Johann Heinrich Hochhuth, a botanist-gardener with extreme attraction to work with entomological material and to collect beetles.

Entomological and other zoological collections of natural history museums allow contemporary researchers to obtain a wide range of information about the species of interest, the historical range of the species, the comparison of the structural features, etc. (Suarez, Tsutsui, 2004). Old museum collections are valued not only by entomologists (Ocheretna, $2019 b$ ) but also biologists of other specialties, including ichthyologists, palaeontologists (Reznick et al., 1994), and archaeozoologists (Hamilton-Dyer, 2013).

Correspondence to: Kateryna Ocheretna; Institute of Zoology, NAS of Ukraine; B. Khmelnytsky St. 15, Kyiv, 01030 Ukraine; e-mail: kateryna_ocheretna@ukr.net; orcid: 0000-0002-7759-8878 
The study is processing data on the collected specimens of the Cryptophagidae family, which are part of J. H. Hochhuth's collection stored in the National Museum of Natural History, NAS of Ukraine, Kyiv. The author of the article worked on biographical information about the collection's author, as well as all the specimens, and also clarified the identification of the specimens.

\section{Biographical information on Johann Hochhuth}

Until recently, information about the researcher was rather incomplete, including absence of it even in B. Mazurmovych's monograph on the milestones of the historical development of zoology in Ukraine (1972), but in 2015 Johann Hochhuth's great-granddaughter published a book containing great deal of information about the researcher's life and work (Hochhuth, 2015). There is also a small essay on J. Hochhuth in the 2012 review on the history of the study of invertebrates in Volhynia (Ivantsiv, Ivantsiv, 2012).

Johann Heinrich Hochhuth (1810-1872) was a talented botanist who made considerable efforts to create the Botanical Garden in Kyiv at St. Volodymyr University (now Taras Shevchenko National University of Kyiv), and also an entomologist whose collections of beetles of the Kyiv region are stored in the National Museum of Natural History, NAS of Ukraine (Kyiv). His scientific articles, devoted mainly to separate families of the Coleoptera, are still used by modern scientists (Fig. 1).

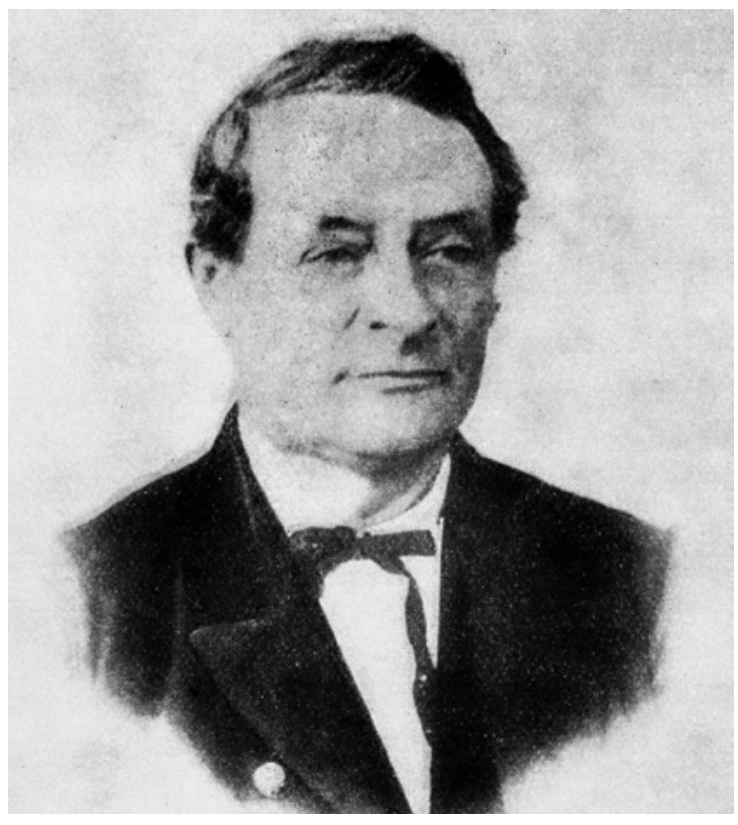

Nowadays we have a slightly different understanding of the term "gardener" of botanical gardens; earlier, it had to be a person with higher education, with knowledge of the basics of agronomy, breeding and a wide range of knowledge in botany in general. Johann Hochhuth was a scientist having exactly those qualities. He was also a gardener of the Botanical Garden and an entomologist who studied and described beetles of the families Staphylinidae and Curculionidae, and wrote articles on general economic topics in Kyiv journals and newspapers, and taught German at the Second Kyiv Gymnasium (Hochhuth, 2015).

Fig. 1. Johann Heinrich Hochhuth, photo from Wikipedia (CC BY-SA 4.0).

Рис. 1. Йоганн Гайнріх Гохгут, фото з інтернет-ресурсу Вікіпедія (CC BY-SA 4.0).

Johann Heinrich was born in 1811 in Kassel city on the Fulda River (Land of Hessesen). After graduating from high school in his hometown, he studied in Vienna. It should be noted that he studied first at the Faculty of Medicine, and then (due to dislike of the anatomy classes) transferred to the Faculty of Biology at the University of Vienna and Tartu (then Derpt). His future mentor, Rudolf Trautfetter, also did the same dropping medicine and diving headlong into botanical science. Johann Hochhuth was interested in botany, entomology, and taxidermy all his life. Some old skin-mounts made by Johann Heinrich are still probably stored at Taras Shevchenko National University of Kyiv (Hochhut, 2015). In 1834, he started to work in Kremenets upon Wilibald Besser's invitation, who was director of the Botanical Garden founded in 1806 at the Kremenets Lyceum.

Two years later, in 1836, after A. L. Andrzejewski's report to the University Council, he requested to provide a job of a gardener and, in conjunction, a taxidermist for Johann Heinrich. Thus, Hochhuth came to Kyiv for the first time, where he was dispatched from Kremenets to St. Volodymyr Imperial University of Kyiv. In 1839, he moved to Kyiv permanently and lived there for the rest of his life. 
In Kyiv, together with Rudolf Trautfetter, Hochhuth developed the Botanical Garden, wrote his finest faunistic works on different groups of beetles, and also kept his collections (Hochhut, 2015).

During his visit to Kyiv, with the permission of the guardian of the Kyiv Educational District, Johann Heinrich brought to St. Volodymyr Imperial University of Kyiv 132 skin-mounts: 108 handstuffed skin-mounts transferred from Hamburg and Vienna, as well as 24 skin-mounts of local species and a bird skeleton.

Johann Heinrich Hochhuth was a member of the Provincial Commission of the Kyiv Educational District (ibid.) and worked in the Botanical Garden of Kyiv University for a long time. In 1850, he was awarded the Gold Medal "For diligent service" (after Nicholas I visited the Botanical Garden in September 1849). During the period of 1849-1873, he published a number of scientific papers, in particular concerning the insect fauna of Ukraine (Hochhuth, 1871, $1872 a-b, 1873$ ).

Entomology was greatly admired by Hochhuth despite his work at the Botanical Garden. He described 60 new species and three new genera of weevils (Hochhuth, $1851 a$ ), and was also interested in the group Staphylinidae (Hochhuth, 1849; $1851 \mathrm{~b}$ ). He amassed a collection of 485 species of Curculionidae of the Kyiv region (Cherkunov, 1889); three species of this family are named after him: Otiorhynchus hochhuthi Marseul, 1872, Melanobaris hochhuthi Faust, 1882, and Phyllobius hochhuthi Faust, 1883. Together with the entomologist Baron Maximilien de Chaudoir, he prepared several scientific works, including a monograph on the beetles of the Caucasus and Transcaucasia (Chaudoir, Hochhuth, 1846), which remains relevant today, and a later article (Hochhuth, 1847).

\section{Overview of the Coleoptera collection}

Johann Heinrich Hochhuth's collection of beetles of the Kyiv region is one of the largest entomological collections (3031 species) described by Mykola Cherkunov (1889). Cherkunov's review contains only a list of beetle names from the collections gathered by J. Hochhuth and Schirmer. Cherkunov's collections, including J. Hochhut's materials, were later transferred to the Pedagogical Museum of Kyiv, and eventually to the National Museum of Natural History, NAS of Ukraine, where they are still preserved as a separate assembly (Zagorodniuk, 2016). Hochhuth's collection of 17285 specimens is catalogued and listed in a database (O. Martynov, pers. comm.), most of the specimens are stored under the original label names. This collection also contains beetles of the Cryptophagidae family, which are the subject of research by the author of this publication. Therefore, they are given special attention.

There are no details regarding the history of this collection, but it can be assumed that the specimens are stored exactly as they were assembled and defined by J. Hochhuth. Some changes might been made later, but considering the names used, this was before M. Cherkunov's review (1889).

\section{The collection of Cryptophagidae species}

Hochhuth's collection of Cryptophagidae includes 214 specimens (Fig. $2 a, b$ ) belonging to 50 species of 10 genera (Fig. 3). Other 4 species (7 specimens) also included in the collection belong to two groups currently recognised as separate families of the Cucujoidea superfamily - the family Languriidae with two species of the genus Leucohimatium (L. arundinaceum (Forskal, 1775) and L. langii [Solsky, 1866]) and one species of Macrophagus (M. robustus Motschulsky, 1845) and family Corylophidae with one species of the genus Orthoperus (O. brunnipes (Gyllenhal, 1808)).

This collection of Cryptophagidae (Tab. 1) was not described before, although some information about its volume was already published (Ocheretna, $2019 a$ ). The history of this collection originates from the entomological collections of the Pedagogical Museum in Kyiv (Zagorodniuk, 2016), collected by various scientists, including J. Hochhuth (1871) and ordered and described by M. Cherkunov (1889).

1 Schirmer was as amateur as J. Hochhuth. According to M. Bilyashivsky, he was a taxidermist at St. Volodymyr Imperial University of Kyiv at the times of K. Kessler. 

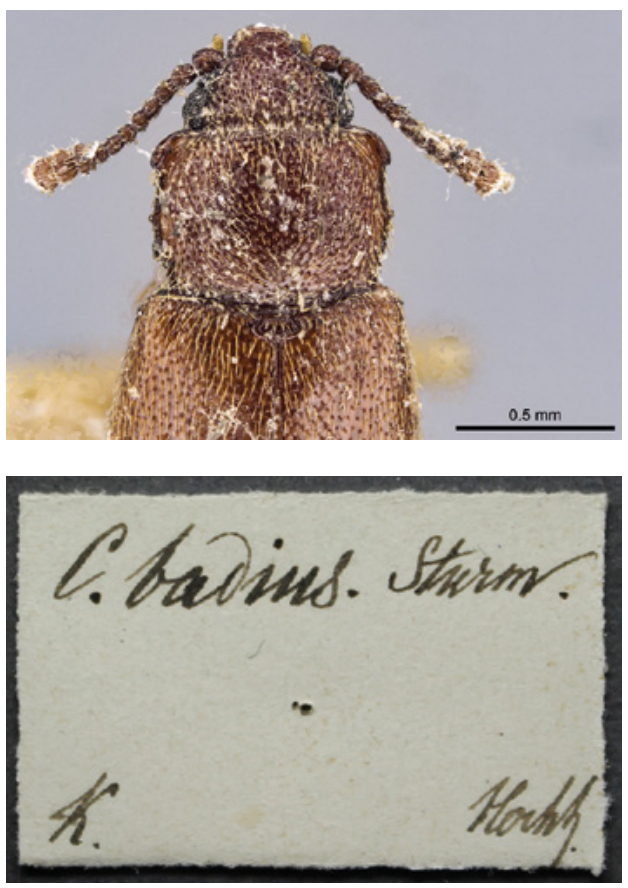

Fig. 2. Cryptophagus badius Sturm, 1845: $a$ - the view of the head and pronotum; $b$ - the original label. Photos by O. Martynov.

Рис. 2. Cryptophagus badius Sturm, 1845: $a$ - вигляд голови та передньоспинки; $b$ - оригінальна етикетка. Фото О. Мартинова.

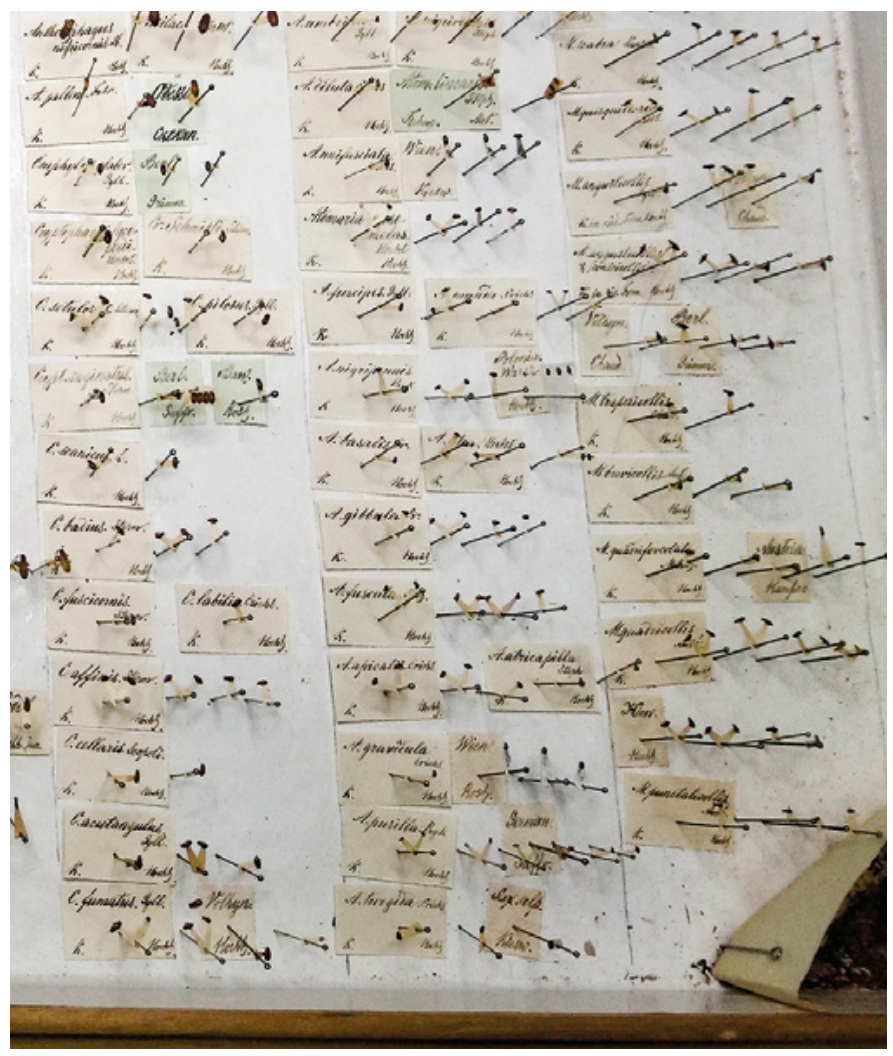

Fig. 3. General view of part of a box with specimens of Cryptophagidae collected by J. H. Hochhuth in the collections of NMNH (Kyiv).

Рис. 3. Загальний вигляд частини ящика зі зразками жуків родини Cryptophagidae, зібраних Й. Г. Гохгутом, у фондах ННПМ (Київ).

Table. 1. The list of species of Cryptophagidae, Languriidae, and Corylophidae families in Hochhuth's collection of beetles

Таблиия 1. Список видів родин Cryptophagidae, Languriidae, Corylophidae серед колекції твердокрилих Й. Г. Гохгута

\begin{tabular}{|c|c|c|c|c|c|}
\hline No. & Label & Valid name & $\begin{array}{l}\text { Number of } \\
\text { specimens }\end{array}$ & Locality and collector & Remarks \\
\hline 1 & $\begin{array}{l}\text { Anterophagus } \\
\text { nigricornis }\end{array}$ & $\begin{array}{l}\text { Anterophagus pallens } \\
\text { (Linnaeus, 1758) }\end{array}$ & 2 & $\begin{array}{l}\text { Kyiv Oblast, Kyiv } \\
\text { Hochhuth J. H. }\end{array}$ & date: unknown \\
\hline 2 & Anterophagus pallens & $\begin{array}{l}\text { Anterophagus pallens } \\
\text { (Linnaeus, 1758) }\end{array}$ & 2 & $\begin{array}{l}\text { Kyiv Oblast, Kyiv } \\
\text { Hochhuth J. H. }\end{array}$ & date: unknown \\
\hline 3 & Anterophagus pallens & $\begin{array}{l}\text { Anterophagus pallens } \\
\text { (Linnaeus, 1758) }\end{array}$ & 1 & $\begin{array}{l}\text { Odesa Oblast, Odesa } \\
\text { O. Czekanowski }\end{array}$ & $\begin{array}{l}\text { date: unknown } \\
\text { Czekan. }\end{array}$ \\
\hline 4 & Antherophagus silaceus & $\begin{array}{l}\text { Antherophagus silaceus } \\
\text { Herbst, } 1792\end{array}$ & 2 & $\begin{array}{l}\text { Kyiv Oblast, Kyiv } \\
\text { Hochhuth J. H. }\end{array}$ & date: unknown \\
\hline 5 & Atomaria diluta & $\begin{array}{l}\text { Atomaria (Agathengis) } \\
\text { diluta Erichson, } 1846\end{array}$ & 1 & $\begin{array}{l}\text { Kyiv Oblast, Kyiv } \\
\text { Hochhuth J. H. }\end{array}$ & date: unknown \\
\hline 6 & Atomaria fimetarii & $\begin{array}{l}\text { Atomaria (Agathengis) } \\
\text { fimetarius (Fabricius, 1792) }\end{array}$ & 3 & $\begin{array}{l}\text { Kyiv Oblast, Kyiv } \\
\text { Hochhuth J. H. }\end{array}$ & date: unknown \\
\hline 7 & Atomaria fimetarii & $\begin{array}{l}\text { Atomaria (Agathengis) } \\
\text { fimetarius (Fabricius, 1792) }\end{array}$ & 2 & $\begin{array}{l}\text { [Germany], Berl.[in] } \\
\text { Grimm B. von }^{1}\end{array}$ & $\begin{array}{l}\text { date: unknown; } \\
\text { Grimm, Berl. }\end{array}$ \\
\hline 8 & Atomaria linearis & $\begin{array}{l}\text { Atomaria (Agathengis) } \\
\text { linearis Stephens, } 1830\end{array}$ & 3 & $\begin{array}{l}\text { loc. unknown } \\
\text { Motschulsky V. }\end{array}$ & $\begin{array}{l}\text { date: unknown; } \\
\text { Motschul. }\end{array}$ \\
\hline 9 & Atomaria nigriventris & $\begin{array}{l}\text { Atomaria (Agathengis) } \\
\text { nigriventris Stephens, } 1830\end{array}$ & 6 & $\begin{array}{l}\text { Kyiv Oblast, Kyiv } \\
\text { Hochhuth J. H. }\end{array}$ & date: unknown \\
\hline 10 & Atomaria umbrina & $\begin{array}{l}\text { Atomaria (Agathengis) } \\
\text { umbrina (Gyllenhal, 1827) }\end{array}$ & 2 & $\begin{array}{l}\text { Kyiv Oblast, Kyiv } \\
\text { Hochhuth J. H. }\end{array}$ & date: unknown \\
\hline
\end{tabular}




\begin{tabular}{|c|c|c|c|c|c|}
\hline No. & Label & Valid name & $\begin{array}{l}\text { Number of } \\
\text { specimens }\end{array}$ & Locality and collector & Remarks \\
\hline 11 & Atomaria apicalis & $\begin{array}{l}\text { Atomaria (Atomaria) } \\
\text { apicalis Erichson, } 1846\end{array}$ & 5 & $\begin{array}{l}\text { Kyiv Oblast, Kyiv } \\
\text { Hochhuth J. H. }\end{array}$ & date: unknown \\
\hline 12 & Atomaria atra & $\begin{array}{l}\text { Atomaria (Atomaria) atra } \\
\text { Herbst, } 1793\end{array}$ & 3 & $\begin{array}{l}\text { Kyiv Oblast, Kyiv } \\
\text { Hochhuth J. H. }\end{array}$ & date: unknown \\
\hline 13 & Atomaria atricapilla & $\begin{array}{l}\text { Atomaria (Atomaria) } \\
\text { atricapilla Stephens, } 1830\end{array}$ & 2 & $\begin{array}{l}\text { Kyiv Oblast, Kyiv } \\
\text { Hochhuth J. H. }\end{array}$ & date: unknown \\
\hline 14 & Atomaria basalis & $\begin{array}{l}\text { Atomaria (Atomaria) basalis } \\
\text { Erichson, } 1846\end{array}$ & 2 & $\begin{array}{l}\text { Kyiv Oblast, Kyiv } \\
\text { Hochhuth J. H. }\end{array}$ & date: unknown \\
\hline 15 & Atomaria fuscata & $\begin{array}{l}\text { Atomaria (Atomaria) fuscata } \\
\text { (Schonherr, 1808) }\end{array}$ & 7 & $\begin{array}{l}\text { Kyiv Oblast, Kyiv } \\
\text { Hochhuth J. H. }\end{array}$ & date: unknown \\
\hline 16 & Atomaria fuscipes & $\begin{array}{l}\text { Atomaria (Atomaria) } \\
\text { fuscipes (Gyllenhal, 1808) }\end{array}$ & 3 & $\begin{array}{l}\text { Kyiv Oblast, Kyiv } \\
\text { Hochhuth J. H. }\end{array}$ & date: unknown \\
\hline 17 & Atomaria gibbula & $\begin{array}{l}\text { Atomaria (Atomaria) gibbula } \\
\text { subsp. gibbula Erichson, } 1846\end{array}$ & 6 & $\begin{array}{l}\text { Kyiv Oblast, Kyiv } \\
\text { Hochhuth J. H. }\end{array}$ & date: unknown \\
\hline 18 & Atomaria gravidula & $\begin{array}{l}\text { Atomaria (Atomaria) } \\
\text { gravidula Erichson, } 1846\end{array}$ & 1 & $\begin{array}{l}\text { Kyiv Oblast, Kyiv } \\
\text { Hochhuth J. H. }\end{array}$ & date: unknown \\
\hline 19 & Atomaria gravidula & $\begin{array}{l}\text { Atomaria (Atomaria) } \\
\text { gravidula Erichson, } 1846\end{array}$ & 3 & $\begin{array}{l}\text { Wien, Austria } \\
\text { Wilhelm Gottlieb } \\
\text { Rosenhauer }\end{array}$ & $\begin{array}{l}\text { date: unknown } \\
\text { Rosh. }\end{array}$ \\
\hline 20 & Atomaria mesomela & $\begin{array}{l}\text { Atomaria (Atomaria) } \\
\text { mesomela (Herbst, 1792) }\end{array}$ & 6 & $\begin{array}{l}\text { Kyiv Oblast, Kyiv } \\
\text { Hochhuth J. H. }\end{array}$ & date: unknown \\
\hline 21 & Atomaria munda & $\begin{array}{l}\text { Atomaria (Atomaria) } \\
\text { munda Erichson, } 1846\end{array}$ & 5 & $\begin{array}{l}\text { Kyiv Oblast, Kyiv } \\
\text { Hochhuth J. H. }\end{array}$ & date: unknown \\
\hline 22 & Atomaria nigripennis & $\begin{array}{l}\text { Atomaria (Atomaria) nigri- } \\
\text { pennis (Kugelann, 1794) }\end{array}$ & 5 & $\begin{array}{l}\text { Kyiv Oblast, Kyiv } \\
\text { Hochhuth J. H. }\end{array}$ & date: unknown \\
\hline 23 & Atomaria nigripennis & $\begin{array}{l}\text { Atomaria (Atomaria) nigri- } \\
\text { pennis (Kugelann, 1794) }\end{array}$ & 3 & Polonia, Warszawa & date: unknown \\
\hline 24 & Atomaria pusilla & $\begin{array}{l}\text { Atomaria (Atomaria) pusilla } \\
\text { (Paykull, 1798) }\end{array}$ & 6 & $\begin{array}{l}\text { Kyiv Oblast, Kyiv } \\
\text { Hochhuth J. H. }\end{array}$ & date: unknown \\
\hline 25 & Atomaria pusilla & $\begin{array}{l}\text { Atomaria (Atomaria) pusilla } \\
\text { (Paykull, 1798) }\end{array}$ & 4 & $\begin{array}{l}\text { German[y] } \\
\text { Christian Wilhelm Lud- } \\
\text { wig Eduard Suffrian }\end{array}$ & $\begin{array}{l}\text { date: unknown } \\
\text { Suffrian }\end{array}$ \\
\hline 26 & Atomaria ruficornis & $\begin{array}{l}\text { Atomaria (Atomaria) } \\
\text { testacea Stephens, } 1830\end{array}$ & 6 & $\begin{array}{l}\text { Kyiv Oblast, Kyiv } \\
\text { Hochhuth J. H. }\end{array}$ & date: unknown \\
\hline 27 & Atomaria testacea & $\begin{array}{l}\text { Atomaria (Atomaria) } \\
\text { testacea Stephens, } 1830\end{array}$ & 3 & $\begin{array}{l}\text { Kyiv Oblast, Kyiv } \\
\text { Hochhuth J. H. }\end{array}$ & date: unknown \\
\hline 28 & Atomaria turgida & $\begin{array}{l}\text { Atomaria (Atomaria) } \\
\text { turgida Erichson, } 1846\end{array}$ & 2 & $\begin{array}{l}\text { Kyiv Oblast, Kyiv } \\
\text { Hochhuth J. H. }\end{array}$ & date: unknown \\
\hline 29 & Atomaria turgida & $\begin{array}{l}\text { Atomaria (Atomaria) } \\
\text { turgida Erichson, } 1846\end{array}$ & 1 & $\begin{array}{l}\text { Germany, Sax. alp. } \\
\text { Kiesn. }\end{array}$ & date: unknown \\
\hline 30 & Atomaria unifasciata & $\begin{array}{l}\text { Atomaria (Atomaria) } \\
\text { unifasciata Erichson, } 1846\end{array}$ & 1 & $\begin{array}{l}\text { Kyiv Oblast, Kyiv } \\
\text { Hochhuth J. H. }\end{array}$ & date: unknown \\
\hline 31 & Atomaria unifasciata & $\begin{array}{l}\text { Atomaria (Atomaria) } \\
\text { unifasciata Erichson, } 1846\end{array}$ & 3 & $\begin{array}{l}\text { Wien, Austria } \\
\text { Kiesn. }\end{array}$ & date: unknown \\
\hline 32 & Atomaria versicolor & $\begin{array}{l}\text { Atomaria (Atomaria) } \\
\text { versicolor Erichson, } 1846\end{array}$ & 1 & $\begin{array}{l}\text { Kyiv Oblast, Kyiv } \\
\text { Hochhuth J. H. }\end{array}$ & date: unknown \\
\hline 33 & $\begin{array}{l}\text { Cryptophagus } \\
\text { acutangulus }\end{array}$ & $\begin{array}{l}\text { Cryptophagus acutangulus } \\
\text { Gyllenhal, } 1828\end{array}$ & 4 & $\begin{array}{l}\text { Kyiv Oblast, Kyiv } \\
\text { Hochhuth J. H. }\end{array}$ & date: unknown \\
\hline 34 & Cryptophagus affinis & $\begin{array}{l}\text { Cryptophagus laticollis } \\
\text { P.H.Lucas, } 1846\end{array}$ & 6 & $\begin{array}{l}\text { Kyiv Oblast, Kyiv } \\
\text { Hochhuth J. H. }\end{array}$ & date: unknown \\
\hline 35 & Cryptophagus badius & $\begin{array}{l}\text { Cryptophagus badius Sturm, } \\
1845\end{array}$ & 4 & $\begin{array}{l}\text { Kyiv Oblast, Kyiv } \\
\text { Hochhuth J. H. }\end{array}$ & date: unknown \\
\hline 36 & Cryptophagus bicolor & $\begin{array}{l}\text { Cryptophagus scutellatus } \\
\text { Newman, } 1834\end{array}$ & 8 & $\begin{array}{l}\text { Kyiv Oblast, Kyiv } \\
\text { Hochhuth J. H. }\end{array}$ & date: unknown \\
\hline 37 & $\begin{array}{l}\text { Cryptophagus } \\
\text { bimaculatus }\end{array}$ & $\begin{array}{l}\text { Micrambe (Micrambinus) } \\
\text { bimaculata (Panzer, 1798) }\end{array}$ & 4 & $\begin{array}{l}\text { Kyiv Oblast, Kyiv } \\
\text { Hochhuth J. H. }\end{array}$ & date: unknown \\
\hline 38 & Cryptophagus cellaris & $\begin{array}{l}\text { Cryptophagus cellaris } \\
\text { (Scopoli, 1763) }\end{array}$ & 2 & $\begin{array}{l}\text { Kyiv Oblast, Kyiv } \\
\text { Hochhuth J. H. }\end{array}$ & date: unknown \\
\hline 39 & Cryptophagus dentatus & $\begin{array}{l}\text { Cryptophagus dentatus } \\
\text { (Herbst, 1793) }\end{array}$ & 1 & $\begin{array}{l}\text { Kyiv Oblast, Kyiv } \\
\text { Hochhuth J. H. }\end{array}$ & date: unknown \\
\hline 40 & Cryptophagus dentatus & $\begin{array}{l}\text { Cryptophagus dent atus } \\
\text { (Herbst, 1793) }\end{array}$ & 1 & $\begin{array}{l}\text { Germany, Berlin } \\
\text { Grimm B. von }{ }^{1}\end{array}$ & $\begin{array}{l}\text { date: unknown; } \\
\text { Grimm, Berl. }\end{array}$ \\
\hline 41 & $\begin{array}{l}\text { Cryptophagus } \\
\text { distinguendus }\end{array}$ & $\begin{array}{l}\text { Cryptophagus distinguendus } \\
\text { Sturm, } 1845\end{array}$ & 1 & $\begin{array}{l}\text { Kyiv Oblast, Kyiv } \\
\text { Hochhuth J. H. }\end{array}$ & date: unknown \\
\hline
\end{tabular}




\begin{tabular}{|c|c|c|c|c|c|}
\hline No. & Label & Valid name & $\begin{array}{l}\text { Number of } \\
\text { specimens }\end{array}$ & Locality and collector & Remarks \\
\hline 42 & Cryptophagus fumatus & $\begin{array}{l}\text { Cryptophagus dentatus } \\
\text { (Herbst, 1793) }\end{array}$ & 4 & $\begin{array}{l}\text { Kyiv Oblast, Kyiv } \\
\text { Hochhuth J. H. }\end{array}$ & date: unknown \\
\hline 43 & Cryptophagus fumatus & $\begin{array}{l}\text { Cryptophagus dentatus } \\
\text { (Herbst, 1793) }\end{array}$ & 2 & $\begin{array}{l}\text { [Volyn Oblast?] } \\
\text { Hochhuth J. H. }\end{array}$ & $\begin{array}{l}\text { date: unknown } \\
\text { Volhyn. }\end{array}$ \\
\hline 44 & $\begin{array}{l}\text { Cryptophagus } \\
\text { fuscicornis }\end{array}$ & $\begin{array}{l}\text { Cryptophagus fuscicornis } \\
\text { Sturm, } 1845\end{array}$ & 1 & $\begin{array}{l}\text { Kyiv Oblast, Kyiv } \\
\text { Hochhuth J. H. }\end{array}$ & date: unknown \\
\hline 45 & Cryptophagus labilis & $\begin{array}{l}\text { Cryptophagus labilis } \\
\text { Erichson, } 1846\end{array}$ & 1 & $\begin{array}{l}\text { Kyiv Oblast, Kyiv } \\
\text { Hochhuth J. H. }\end{array}$ & date: unknown \\
\hline 46 & Cryptophagus sp. & $\begin{array}{l}\text { Cryptophagus laticollis } \\
\text { P. H. Lucas, } 1846\end{array}$ & 1 & $\begin{array}{l}\text { [Russian Federation?], } \\
\text { Kazan } \\
\text { Ballion E. von }{ }^{3}\end{array}$ & $\begin{array}{l}\text { date: unknown } \\
\text { Ballion, Kazan } \\
\text { id. K. Ocheretna }\end{array}$ \\
\hline 47 & Cryptophagus lycoperdi & $\begin{array}{l}\text { Cryptophagus lycoperdi } \\
\text { (Scopoli, 1763) }\end{array}$ & 1 & $\begin{array}{l}\text { Kyiv Oblast, Kyiv } \\
\text { Hochhuth J. H. }\end{array}$ & date: unknown \\
\hline 48 & Cryptophagus pilosus & $\begin{array}{l}\text { Cryptophagus pilosus } \\
\text { Gyllenhal, } 1827\end{array}$ & 1 & $\begin{array}{l}\text { Kyiv Oblast, Kyiv } \\
\text { Hochhuth J. H. }\end{array}$ & date: unknown \\
\hline 49 & Cryptophagus populi & $\begin{array}{l}\text { Cryptophagus populi } \\
\text { Paykull, } 1800\end{array}$ & 4 & $\begin{array}{l}\text { Kyiv Oblast, Kyiv } \\
\text { Hochhuth J. H. }\end{array}$ & date: unknown \\
\hline 50 & $\begin{array}{l}\text { Cryptophagus } \\
\text { pubescens }\end{array}$ & $\begin{array}{l}\text { Cryptophagus pubescens } \\
\text { Sturm, } 1845\end{array}$ & 6 & $\begin{array}{l}\text { Kyiv Oblast, Kyiv } \\
\text { Hochhuth J. H. }\end{array}$ & date: unknown \\
\hline 51 & Cryptophagus saginatus & $\begin{array}{l}\text { Cryptophagus saginatus } \\
\text { Sturm, } 1845\end{array}$ & 2 & $\begin{array}{l}\text { Kyiv Oblast, Kyiv } \\
\text { Hochhuth J. H. }\end{array}$ & date: unknown \\
\hline 52 & Cryptophagus saginatus & $\begin{array}{l}\text { Cryptophagus saginatus } \\
\text { Sturm, } 1845\end{array}$ & 4 & $\begin{array}{l}\text { [Germany], Berl.[in] } \\
\text { Christian Wilhelm Lud- } \\
\text { wig Eduard Suffrian }\end{array}$ & $\begin{array}{l}\text { date: unknown } \\
\text { Suffrian }\end{array}$ \\
\hline 53 & Cryptophagus saginatus & $\begin{array}{l}\text { Cryptophagus saginatus } \\
\text { Sturm, } 1845\end{array}$ & 1 & $\begin{array}{l}\text { [Germany], Baw.[aria] } \\
\text { Wilhelm Gottlieb } \\
\text { Rosenhauer }\end{array}$ & $\begin{array}{l}\text { date: unknown } \\
\text { Rosh. }\end{array}$ \\
\hline 54 & Cryptophagus scanicus & $\begin{array}{l}\text { Cryptophagus scanicus } \\
\text { (Linnaeus, 1758) }\end{array}$ & 2 & $\begin{array}{l}\text { Kyiv Oblast, Kyiv } \\
\text { Hochhuth J. H. }\end{array}$ & date: unknown \\
\hline 55 & Cryptophagus schmidti & $\begin{array}{l}\text { Cryptophagus schmidti } \\
\text { Sturm, } 1845\end{array}$ & 1 & $\begin{array}{l}\text { Kyiv Oblast, Kyiv } \\
\text { Hochhuth J. H. }\end{array}$ & date: unknown \\
\hline 56 & Cryptophagus setulosus & $\begin{array}{l}\text { Cryptophagus setulosus } \\
\text { Sturm, } 1845\end{array}$ & 6 & $\begin{array}{l}\text { Kyiv Oblast, Kyiv } \\
\text { Hochhuth J. H. }\end{array}$ & date: unknown \\
\hline 57 & Cryptophagus vini & $\begin{array}{l}\text { Micrambe ulicis (Stephens, } \\
\text { 1830) }\end{array}$ & 3 & $\begin{array}{l}\text { Kyiv Oblast, Kyiv } \\
\text { Hochhuth J. H. }\end{array}$ & date: unknown \\
\hline 58 & Ephistemus exiguus & $\begin{array}{l}\text { Curelius exiguus (Erichson, } \\
1846 \text { ) }\end{array}$ & 2 & $\begin{array}{l}\text { Kyiv Oblast, Kyiv } \\
\text { Hochhuth J. H. }\end{array}$ & date: unknown \\
\hline 59 & Ephistemus exiguus & $\begin{array}{l}\text { Curelius exiguus (Erichson, } \\
\text { 1846) }\end{array}$ & 7 & $\begin{array}{l}\text { Kyiv Oblast, Kyiv } \\
\text { Hochhuth J. H. }\end{array}$ & date: unknown \\
\hline 60 & Ephistemus gyrinoides & $\begin{array}{l}\text { Ephistemus globulus } \\
\text { (Paykull, 1798) }\end{array}$ & 6 & $\begin{array}{l}\text { Kyiv Oblast, Kyiv } \\
\text { Hochhuth J. H. }\end{array}$ & date: unknown \\
\hline 61 & $\begin{array}{l}\text { Ephistemus gyrinoides } \\
\text { var. dimidiatus }\end{array}$ & $\begin{array}{l}\text { Ephistemus globulus } \\
\text { (Paykull, 1798) }\end{array}$ & 2 & $\begin{array}{l}\text { Kyiv Oblast, Kyiv } \\
\text { Hochhuth J. H. }\end{array}$ & date: unknown \\
\hline 62 & $\begin{array}{l}\text { Ephistemus gyrinoides } \\
\text { var. dimidiatus }\end{array}$ & $\begin{array}{l}\text { Ephistemus globulus } \\
\text { (Paykull, 1798) }\end{array}$ & 2 & $\begin{array}{l}\text { German[y] } \\
\text { Christian Wilhelm Lud- } \\
\text { wig Eduard Suffrian }\end{array}$ & $\begin{array}{l}\text { date: unknown } \\
\text { Suffrian }\end{array}$ \\
\hline 63 & Ephistemus globulus & $\begin{array}{l}\text { Ephistemus globulus } \\
\text { (Paykull, 1798) }\end{array}$ & 6 & $\begin{array}{l}\text { Kyiv Oblast, Kyiv } \\
\text { Hochhuth J. H. }\end{array}$ & date: unknown \\
\hline 64 & Paramecosoma abietis & $\begin{array}{l}\text { Micrambe abietis (Paykull, } \\
1798 \text { ) }\end{array}$ & 1 & $\begin{array}{l}\text { Kyiv Oblast, Kyiv } \\
\text { Hochhuth J. H. }\end{array}$ & $\begin{array}{l}\text { date: unknown } \\
\text { under Form.acerd. }\end{array}$ \\
\hline 65 & $\begin{array}{l}\text { Paramecosoma } \\
\text { serratum }\end{array}$ & $\begin{array}{l}\text { Henoticus serratus } \\
\text { (Gyllenhal, 1808) }\end{array}$ & 2 & $\begin{array}{l}\text { Kyiv Oblast, Kyiv } \\
\text { Hochhuth J. H. }\end{array}$ & date: unknown \\
\hline 66 & $\begin{array}{l}\text { Paramecosoma } \\
\text { serratum }\end{array}$ & $\begin{array}{l}\text { Henoticus serratus } \\
\text { (Gyllenhal, 1808) }\end{array}$ & 2 & $\begin{array}{l}\text { Fennia [Finland] } \\
\text { mer Mähl }\end{array}$ & date: unknown \\
\hline 67 & $\begin{array}{l}\text { Paramecosoma } \\
\text { melanocephalum }\end{array}$ & $\begin{array}{l}\text { Paramecosoma melanoce- } \\
\text { phalum (Herbst, 1793) }\end{array}$ & 2 & $\begin{array}{l}\text { Kyiv Oblast, Kyiv } \\
\text { Hochhuth J. H. }\end{array}$ & date: unknown \\
\hline 68 & Emphylus glaber & $\begin{array}{l}\text { Spavius glaber (Gyllenhal, } \\
1808 \text { ) }\end{array}$ & 1 & $\begin{array}{l}\text { Kyiv Oblast, Kyiv } \\
\text { Hochhuth J. H. }\end{array}$ & date: unknown \\
\hline 69 & Emphylus glaber & $\begin{array}{l}\text { Spavius glaber (Gyllenhal, } \\
1808 \text { ) }\end{array}$ & 2 & $\begin{array}{l}\text { [Germany], Berl.[in] } \\
\text { Grimm B. von }{ }^{1}\end{array}$ & $\begin{array}{l}\text { date: unknown; } \\
\text { Grimm, Berl. }\end{array}$ \\
\hline 70 & Telmatophilus caricis & $\begin{array}{l}\text { Telmatophilus caricis } \\
\text { (Olivier, 1790) }\end{array}$ & 1 & $\begin{array}{l}\text { reg. unknown } \\
\text { O. Czekanowski }\end{array}$ & $\begin{array}{l}\text { date: unknown } \\
\text { Czekan. Pod A. }\end{array}$ \\
\hline 71 & Telmatophilus caricis & $\begin{array}{l}\text { Telmatophilus caricis } \\
\text { (Olivier, 1790) }\end{array}$ & 2 & $\begin{array}{l}\text { Kyiv Oblast, Kyiv } \\
\text { Hochhuth J. H. }\end{array}$ & date: unknown \\
\hline
\end{tabular}




\begin{tabular}{|c|c|c|c|c|c|}
\hline No. & Label & Valid name & $\begin{array}{l}\text { Number of } \\
\text { specimens }\end{array}$ & Locality and collector & Remarks \\
\hline 72 & Telmatophilus typhae & $\begin{array}{l}\text { Telmatophilus typhae } \\
\text { (Fallen, 1802) }\end{array}$ & 3 & $\begin{array}{l}\text { Kyiv Oblast, Kyiv } \\
\text { Hochhuth J. H. }\end{array}$ & date: unknown \\
\hline \multicolumn{6}{|c|}{ Languriidae } \\
\hline 73 & $\begin{array}{l}\text { Leucohimatium } \\
\text { elongatum }\end{array}$ & $\begin{array}{l}\text { Leucohimatium } \\
\text { arundinaceum (Forskal, } \\
\text { 1775) }\end{array}$ & 1 & $\begin{array}{l}\text { reg. unknown } \\
\text { Kiesn.[er?] }\end{array}$ & $\begin{array}{l}\text { date: unknown } \\
\text { Actol }\end{array}$ \\
\hline 74 & Paramecosoma langii & $\begin{array}{l}\text { Leucohimatium langii } \\
\text { (Solsky, 1866) }\end{array}$ & 1 & $\begin{array}{l}\text { Kyiv Oblast, Kyiv } \\
\text { Hochhuth J. H. }\end{array}$ & date: unknown \\
\hline 75 & Haplolophus robustus & $\begin{array}{l}\text { Macrophagus robustus } \\
\text { Motschulsky, } 1845\end{array}$ & 2 & $\begin{array}{l}\text { Kyiv Oblast, Kyiv } \\
\text { Hochhuth J. H. }\end{array}$ & date: unknown \\
\hline \multicolumn{6}{|c|}{ Corylophidae } \\
\hline 76 & Ephistemus nigriclavis & $\begin{array}{l}\text { Orthoperus brunnipes } \\
\text { (Gyllenhal, 1808) }\end{array}$ & 3 & $\begin{array}{l}\text { Kyiv Oblast, Kyiv } \\
\text { Hochhuth J. H. }\end{array}$ & date: unknown \\
\hline
\end{tabular}

The collection contains specimens mainly from the Kyiv region from the middle of the 19th century. In addition to J. Hochhuth's own collections (180 specimens), it contains specimens collected by other entomologists, including W. Rosengauer (Austria and Germany: 4 spec., 2 species), K. Suffrian (Germany: 10 spec., 3 species), E. Ballion (Russia: 1 spec., 1 species), B. Grimm (Germany: 5 spec., 3 species), O. Czekanowski (Ukraine: 2 spec., 2 species), and others.

In general, the collection includes three unique species that are not represented in any other Ukrainian entomological collections examined by the author (Ocheretna, $2019 a$ ). These species are Atomaria basalis Erichson, 1846 (2 spec.), A. versicolor Erichson, 1846 (1 spec.), and Cryptophagus labilis Erichson, 1846 (1 spec.). All specimens were collected in Kyiv. It should be also noted that after J. Hochhuth these species did not occur in the collections of natural history museums and in the author's own collections from the territory of Ukraine.

\section{The significance of the collection}

The collection is extremely valuable and important for the study of morphological features of individual species of the family in comparison with modern specimens, as well as for the accumulation of data on changes of the Cryptophagidae fauna in Ukraine. Unfortunately, the overwhelming majority of the specimens do not contain the date of collection on the labels, and we only know about the approximate period of collecting (often the researcher's years of work, not exactly). Nevertheless, the value of the specimens is undoubted, since, given the present, such accuracy of dating is sufficient.

The collection is also important for studying the history of formation of modern large zoological collections, particularly because it passed a difficult way from J. Hochhuth to M. Cherkunov's collection, then to the natural history collection of the Pedagogical Museum and, finally, to the collections of the National Museum of Natural History, NAS of Ukraine.

The materials of this collection (taking into account the re-identifications and ordering the nomenclature to modern standards) represent an important and complete set of Cryptophagidae species that occurred in the territory of Kyiv and Kyiv region in the middle of the 19th century.

\section{References}

Chaudoir, M. de, J. H. Hochhuth. 1846. Enumération des carabiques et hydrocanthares: recueillis pendant un voyage au Caucase et dans les provinces transcaucasiennes par le baron M. de Chaudoir et le baron A. de Gotsch; carabiques. Kiew, 1-268.

Cherkunov, M. 1889. The list of beetles in Kyiv and it's outskirts. Notes of the Kyiv Society of Naturalists, 10 (1): 147-204.

Hamilton-Dyer, S. 2013. The Reference Collection — Is it Dead? The Role of the Physical Reference Collection in the Digital Age. Archaeofauna, 22 (1): 75-82.

Hochhuth, J.H. 1847. Enumeration der Rüsselkäfer, welche vom Baron Maximilian v. Chaudoir und vom Baron A. v. Gotsch auf ihren Reisen im Kaukasus und in Transkaukasien im Jahre 1845... Arten. Bull. Soc. Nat. Moscou, 20 (1): 448-587.

Hochhuth, J. H. 1849. Die Staphylinen-Fauna des Kaukasus und Transkaukasien. Bull. Soc. Nat. Moscou, 22 (1): $18-214$. 
Hochhuth, J. H. 1851 a. Beitraege zur näheren Kenntniss der Rüsselkäfer Russlands. Enthaltend Beschreibung neuer Genera und Arten, nebst Erläurerungen noch nicht hinlänglich bekannter Curculionen des Russischen Reichs. Bull. Soc. Nat. Moscou, 24 (1): 3-102.

Hochhuth, J. H. 1851 b. Beitraege zur näheren Kenntniss der Staphylinen Russlands. Enthaltend Beschreibung neuer Genera und Arten, nebst Erläurerungen noch nicht hinlänglich bekannter Staphylinen des Russischen Reichs. Bull. Soc. Nat. Moscou, 24 (2): 3-58.

Hochhuth, J. H. 1871. Enumeration der in der russischen Gouvemement Kiew und Wolhynien bisher aufgefundenen Käfer. Bull. Soc. Nat. Moscou, 44 (1-2): 176-253.

Hochhuth, J. H. 1872 a. Enumeration der in den russischen gouvernements Kiew und Volhynien bisher aufgefundenen Käfer. Bull. Soc. Nat. Moscou, 44 (3-4): 85-177.

Hochhuth, J. H. 1872 b. Enumeration der in den russischen gouvernements Kiew und Volhynien bisher aufgefundenen Käfer. Bull. Soc. Nat. Moscou, 45 (3): 195-234.

Hochhuth, J. H. 1873. Enumeration der in den russischen Gouvernements Kiew und Volhynien bisher augefundenen Käfer. Bull. Soc. Nat. Moscou, 46: 124-164.

Hochhuth, T. N. 2015. I do not say with longing they are not here, but with pride that they were. Economic review of the Kiev province of 1845. Sputnik Plus, Moscow, 1-269.

Ivantsiv, V. V., O. Ya. Ivantsiv. 2012. The study of fauna of invertebrata grouhs on the territory of Shatsk Lakes (of XIX century). Nature of Western Polissia and surrounding areas, 9 (2): 264-269.

Mazurmovych, B. M. 1972. The development of zoology in Ukraine. Kyiv University Publishing House, Kyiv, $1-229$.

Ocheretna, K. 2019 a. Cryptophagidae (Coleoptera) in the collections of Ukraine: species, specimens, and collectors. Proceedings of the State Museum of Natural History, 35: 21-36.

Ocheretna, K. 2019 b. Cryptophagidae (Coleoptera) in Volodymyr Lazorko's collection stored at Schmalhausen Institute of Zoology (Kyiv, Ukraine). GeoßBio, 17: 62-76.

Ocheretna, K. 2019 c. Naturalist Johann Heinrich Hochhuth and his collection of Cryptophagidae (Coleoptera: Cucujoidea). In: I. Zagorodniuk (Ed.). Natural History Museology (Natural History Museums in Ukraine: Formation and Prospects for Development). Vol. 5. NMNH NAS of Ukraine, Kyiv, 78-81.

Reznick, D., R. J. Baxter, J. Endler. 1994. Long-term Studies of Tropical Stream Fish Communities: The Use of Field Notes and Museum Collections to Reconstruct Communities of the Past. Integrative and Comparative Biology, 34 (3): 452-462.

Suarez, A. V., N. D. Tsutsui. 2004. The Value of Museum Collections for Research and Society, BioScience, 54 (1): 66-74.

Zagorodniuk, I. 2016. Natural History Collections in the Kyiv Pedagogical Museum in 1902-1917. Proceedings of the National Museum of Natural History, 14: 123-135. 